The Nepal Math. Sc. Report

Vol. 36, No.1 and 2, 2019

\title{
FATOU, JULIA, AND ESCAPING SETS OF CONJUGATE HOLOMORPHIC SEMIGROUPS
}

\author{
BISHNU HARI SUBEDI ${ }^{1}$ AND AJAYA SINGH ${ }^{2}$ \\ ${ }^{1,2}$ Central Department of Mathematics, Institute of Science and Technology, Tribhuvan \\ University, Kirtipur, Kathmandu, Nepal \\ Email: subedi.abs@gmail.com,singh.ajaya1@gmail.com
}

\begin{abstract}
We define commutator of a holomorphic semigroup, and on the basis of this concept, we define conjugate semigroups of a holomorphic semigroup. We prove that the conjugate semigroup is nearly abelian if and only if the given holomorphic semigroup is nearly abelian. We also prove that image of each of Fatou, Julia, and escaping sets of a holomorphic semigroup under commutator (affine complex conjugating map) is equal respectively, to the Fatou, Julia, and escaping sets of the conjugate semigroup. Finally, we prove that every element of a nearly abelian holomorphic semigroup $S$ can be written as the composition of an element from the set generated by the set of commutators $\Phi(S)$ and the composition of the certain powers of its generators..
\end{abstract}

Key Words: Holomorphic semigroup, nearly abelian semigroup, commutator, conjugate semigroup.

AMS (MOS) [2010] Subject Classification. 37F10, 30D05

\section{INTRODUCTION}

We confine our study on Fatou, Julia, and escaping sets of holomorphic semigroups and their conjugate semigroups. It is very obvious fact that a set of holomorphic functions naturally forms a semigroup. Here, we take a set $A$ of holomorphic functions and construct a semigroup $S$ consists of all elements that can be expressed as a finite composition of elements in $A$. We call such a semigroup $S$ by holomorphic semigroup generated by the set $A$. Our particular interest is to study of the dynamics of the families of holomorphic functions. For a collection $\mathscr{F}=\left\{f_{\alpha}\right\}_{\alpha \in \Delta}$ of such functions, let

$$
S=\left\langle f_{\alpha}\right\rangle
$$

be a holomorphic semigroup generated by them. The index set $\Delta$ to which $\alpha$ belongs is allowed to be infinite in general unless otherwise stated. Here, each $f \in S$ is a holomorphic function and $S$ is closed under functional composition. Thus, $f \in S$ is constructed through the composition of finite number of functions $f_{\alpha_{k}},(k=1,2,3, \ldots, m)$. That is,

$$
f=f_{\alpha_{1}} \circ f_{\alpha_{2}} \circ f_{\alpha_{3}} \circ \cdots \circ f_{\alpha_{m}} .
$$

This research work of the first author is supported by a PhD faculty fellowship of the University Grants Commission, Nepal. 
A semigroup generated by finitely many holomorphic functions $f_{i},(i=1,2, \ldots, n)$ is called finitely generated holomorphic semigroup and we write it by

$$
S=\left\langle f_{1}, f_{2}, \ldots, f_{n}\right\rangle \text {. }
$$

. The holomorphic semigroup $S$ is abelian if

$$
f_{\alpha} \circ f_{\beta}=f_{\beta} \circ f_{\alpha}
$$

for all $\alpha, \beta \in\{\alpha: \alpha \in \Delta\}$. The semigroup $S$ is right cancellative if

$$
f \circ g=h \circ g \Longrightarrow f=h,
$$

and left cancellative if

$$
h \circ g=h \circ f \Longrightarrow g=f
$$

for all $f, g, h \in S$ and cancellative if it is both right and left cancellative. The family $\mathscr{F}$ of holomorphic functions forms a normal family in a domain $D$ if given any composition sequence $\left(f_{\alpha}\right)$ generated by the member of $\mathscr{F}$, there is a subsequence $\left(f_{\alpha_{k}}\right), \alpha_{k} \in\{\alpha: \alpha \in$ $\Delta\}$, which is uniformly convergent or divergent on all compact subsets of $D$. If there is a neighborhood $U$ of the point $z \in \mathbb{C}$ such that $\mathscr{F}$ is normal family in $U$, then we say $\mathscr{F}$ is normal at $z$. If $\mathscr{F}$ is a semigroup $S$, then we simply say that $S$ is normal in the neighborhood of $z$ or $S$ is normal at $z$. Semigroup $S$ is iteratively divergent at $z$ if $f^{n}(z) \rightarrow \infty$ as $n \rightarrow \infty$ for all $f \in S$.

Based on the Fatou-Julia-Eremenko theory of a holomorphic function, the Fatou, Julia, and escaping sets in the settings of transcendental semigroup are defined as follows.

Definition 1.1 (Fatou, Julia, and escaping sets). Fatou set of the transcendental semigroup $S$ is defined by

$$
F(S)=\{z \in \mathbb{C}: S \text { is normal in a neighborhood of } z\}
$$

and the Julia set $J(S)$ of $S$ is the compliment of $F(S)$ where as the escaping set of $S$ is defined by

$$
I(S)=\{z \in \mathbb{C}: S \text { is iteratively divergent at } z\} .
$$

We call each point of the set $I(S)$ by an escaping point.

There is a slightly larger family of holomorphic semigroups that can fulfill most of the results of abelian holomorphic semigroups. We call these semigroups nearly abelian and it is considered the more general form than that of abelian semigroups.

Definition 1.2 (Nearly abelian semigroup). We say that a holomorphic semigroup $S$ is nearly abelian if there is a family $\Phi=\left\{\phi_{i}\right\}$ of conformal maps such that

(1) $\phi_{i}(F(S))=F(S)$ for all $\phi_{i} \in \Phi$ and

(2) for all $f, g \in S$, there is a $\phi \in \Phi$ such that $f \circ g=\phi \circ g \circ f$.

The set $\Phi$ of Definition 1.2 has given a special name as define below. 
Definition 1.3 (Commutator). Let $S$ be a holomorphic semigroup. The set of the form

$$
\Phi(S)=\{\phi: \text { there are } f, g \in S \text { such that } f \circ g=\phi \circ g \circ f\}
$$

is called the set of commutators of $S$. We write $\phi=[f, g]$ if $f \circ g=\phi \circ g \circ f$.

The notion of commutator is very useful to obtain conjugate maps of each generator $f_{i}$ of the semigroup $S$ and conjugate semigroup of the semigroup $S$.

Definition 1.4 (Conjugate semigroup). Let $S=\left\langle f_{1}, f_{2}, f_{3}, \ldots, f_{n}\right\rangle$ be a finitely generated holomorphic semigroup and $\Phi(S)$ be a set of its commutators. Let us define a set

$$
S^{\prime}=\left\langle\phi \circ f_{1} \circ \phi^{-1}, \phi \circ f_{2} \circ \phi^{-1}, \ldots, \phi \circ f_{n} \circ \phi^{-1}\right\rangle
$$

where $\phi \in \Phi(S)$ such that $\phi=\left[f_{i}, f_{j}\right]$ and $\phi^{-1}=\left[f_{j}, f_{i}\right]$ as we defined before. If we let $g_{i}=\phi \circ f_{i} \circ \phi^{-1}$, then we say function $f_{i}$ is conjugate to $g_{i}$ by a map $\phi: \mathbb{C} \rightarrow \mathbb{C}$. The semigroup $S^{\prime}$ is then called a conjugate semigroup of the semigroup $S$.

The image of the Fatou, Julia, and escaping sets of a nearly abelian semigroup under commutator $\phi \in \Phi(S)$ is respectively equal to the Fatou, Julia, and escaping sets of its conjugate.

Theorem 1.1. Let $S=\left\langle f_{1}, f_{2}, f_{3}, \ldots, f_{n}\right\rangle$ be a nearly abelian holomorphic semigroup and $\Phi$ be a set of commutators of the form $\phi(z)=a z+b$ for some non-zero a. Let $S^{\prime}$ be a conjugate semigroup of $S$. Then there is a $\phi \in \Phi$ such that $\phi(F(S))=F\left(S^{\prime}\right), \phi(I(S))=I\left(S^{\prime}\right)$ and $\phi(J(S))=J\left(S^{\prime}\right)$.

We generalize [1, Theorem 4.3] to the following result.

Theorem 1.2. Let $S=\left\langle f_{1}, f_{2}, f_{3}, \ldots, f_{n}, \ldots\right\rangle$ be a nearly abelian cancellative holomorphic semigroup and $\Phi(S)$ be a set of commutators of $S$. Then for every $f \in S$, we have

$$
f=\phi \circ f_{1}^{t_{1}} \circ f_{2}^{t_{2}} \circ f_{3}^{t_{3}} \circ \cdots f_{m}^{t_{m}}
$$

where $\phi \in \Phi(S)$ if $\Phi(S)$ is a group or semigroup otherwise $\phi \in G$, where $G=\langle\Phi(S)\rangle$ is a group generated by $\Phi(S)$ and $t_{i}$ are non-negative integers.

\section{The Notion of Conjugate Semigroup and the Proof of Theorem 1.1}

Let $S$ be a holomorphic semigroup. If there is a holomorphic function $\phi$ such that $f \circ g=\phi \circ g \circ f$ for every pair of functions $f, g \in S$, then $\phi$ is called commutator of $f$ and $g$. Note that such a commutator is unique for every pair of holomorphic functions. Recall that

$$
\Phi(S)=\{\phi: f \circ g=\phi \circ g \circ f \text { for every pair of functions } f, g \in S\}
$$

is a set of commutators of holomorphic semigroup $S$. If $S$ is abelian, then commutator $\phi$ is an identity function. By Definition 1.3, we write $\phi=[f, g]$ if $f \circ g=\phi \circ g \circ f$. Note that $[f, g]^{-1}=[g, f]$ and for any $f \in S,[f, f]=$ identity. So, in $\Phi(S)$, there is an identity element and inverse of each $\phi \in \Phi(S)$. It is not clear in general whether $\Phi(S)$ has group structure or semigroup structure but we can make a group or semigroup $G=\langle\Phi(S)\rangle$ generated by the 
elements of $\Phi(S)$ whenever it is necessary. Note that there are some commutator identities of groups which can be verified in $\Phi(S)$ if $S$ is a cancellative holomorphic semigroup. For example:

(1) $\left[f, g \circ f^{n}\right]=[f, g]$.

(2) $\left[f, f^{n} \circ g\right] \circ f^{n}=f^{n} \circ[f, g]$.

(3) $[f \circ g, g \circ f] \circ g \circ f=f \circ g \circ[g, f]$

In practice, there is a commutator for given pair of holomorphic functions.

Example 2.1. Let $f(z)=\exp z^{2}+\lambda$ and $g(z)=-f(z)$, where $\lambda \in \mathbb{C}$. It is easy to see that

$$
(f \circ g)(z)=\exp \left(\exp z^{2}+\lambda\right)^{2}+\lambda=\phi\left(-\exp \left(\exp z^{2}+\lambda\right)^{2}-\lambda\right)=(\phi \circ g \circ f)(z),
$$

where $\phi(z)=-z$. Likewise, if $f(z)=\lambda \cos z,(\lambda \in \mathbb{C})$ and $g(z)=-f(z)$, then

$$
(f \circ g)(z)=\lambda \cos (\lambda \cos z)=\phi(-\lambda \cos (\lambda \cos z))=(\phi \circ g \circ f)(z), \text { where } \phi(z)=-z
$$

We prove the following result that shows conjugate semigroup is nearly abelian if and only if the holomorphic semigroup itself is nearly abelian.

Theorem 2.1. Let $S^{\prime}$ be a conjugate semigroup of a holomorphic semigroup $S$. Then $S^{\prime}$ is nearly abelian if and only if $S$ is nearly abelian.

Proof. Let $S=\left\langle f_{1}, f_{2}, f_{3}, \ldots, f_{n}\right\rangle$ be a nearly abelian holomorphic semigroup and

$$
S^{\prime}=\left\langle\phi \circ f_{1} \circ \phi^{-1}, \phi \circ f_{2} \circ \phi^{-1}, \ldots, \phi \circ f_{n} \circ \phi^{-1}\right\rangle
$$

be the conjugate semigroup of $S$ where $\phi \in \Phi(S)$. Then, there is a $\phi \in \Phi(S)$ such that $f_{i} \circ f_{j}=\phi \circ f_{j} \circ f_{i}$ for all generators $f_{i}, f_{j} \in S$. Now for any $\phi \circ f_{i} \circ \phi^{-1}, \phi \circ f_{j} \circ \phi^{-1} \in S^{\prime}$, we have

$$
\begin{aligned}
\left(\phi \circ f_{i} \circ \phi^{-1}\right) \circ\left(\phi \circ f_{j} \circ \phi^{-1}\right) & =\phi \circ f_{i} \circ f_{j} \circ \phi^{-1} \\
& =\phi \circ \xi \circ f_{j} \circ f_{i} \circ \phi^{-1} \text { for some } \xi \in \Phi(S) \\
& =\xi \circ \phi \circ f_{j} \circ f_{i} \circ \phi^{-1} \\
& =\xi \circ\left(\phi \circ f_{j} \circ \phi^{-1}\right) \circ\left(\phi \circ f_{i} \circ \phi^{-1}\right)
\end{aligned}
$$

This shows that $S^{\prime}$ is nearly abelian.

Conversely, suppose that semigroup $S^{\prime}$ is nearly abelian. Then, there is $\phi \in \Phi(S)$ such that $g_{i} \circ g_{j}=\phi \circ g_{j} \circ g_{i}$, where $g_{i}=\phi \circ f_{i} \circ \phi^{-1}$ and $g_{j}=\phi \circ f_{j} \circ \phi^{-1}$ and from which get $f_{i}=\phi^{-1} \circ g_{i} \circ \phi$ and $f_{j}=\phi^{-1} \circ g_{j} \circ \phi$. Now, for any $f_{i}, f_{j} \in S$, we have

$$
\begin{aligned}
f_{i} \circ f_{j} & =\left(\phi^{-1} \circ g_{i} \circ \phi\right) \circ\left(\phi^{-1} \circ g_{j} \circ \phi\right) \\
& =\phi^{-1} \circ g_{i} \circ g_{j} \circ \phi \\
& =\phi^{-1} \circ \phi \circ g_{j} \circ g_{i} \circ \phi \\
& =g_{j} \circ g_{i} \circ \phi \\
& =\phi \circ f_{j} \circ \phi^{-1} \circ \phi \circ f_{i} \circ \phi^{-1} \circ \phi \\
& =\phi \circ f_{j} \circ f_{i}
\end{aligned}
$$


This shows that $S$ is nearly abelian.

Theorem 2.1 tells us that if there is a nearly abelian holomorphic semigroup, then we can construct other nearly abelian semigroups by making conjugate semigroups. To prove Theorem 1.1, we need the following lemma.

Lemma 2.1. Let $f$ and $g$ be two holomorphic functions and $\phi$ be an entire function of the form $z \rightarrow a z+b$, where $a \neq 0$ such that $\phi \circ f=g \circ \phi$. Then $\phi(I(f))=I(g), \phi(J(f))=J(g)$ and $\phi(F(f))=F(g)$.

Proof. Let $w \in \phi(I(f))$, then there is $z \in I(f)$ such that $w=\phi(z)$. The condition $z \in$ $I(f) \Longrightarrow f^{n}(z) \rightarrow \infty$ as $n \rightarrow \infty$. Now, $g^{n}(w)=g^{n}(\phi(z))=\left(g^{n} \circ \phi\right)(z)=\left(g^{n-1} \circ g \circ\right.$ $\phi)(z)=\left(g^{n-1} \circ \phi \circ f\right)(z)=\left(g^{n-2} \circ \phi \circ f^{2}\right)(z)=\ldots=\left(\phi \circ f^{n}\right)(z)=\phi\left(f^{n}(z)\right)$. Since $\phi(z)=a z+b,(a \neq 0)$ and $f^{n}(z) \rightarrow \infty$ as $n \rightarrow \infty$, so we must have $g^{n}(w) \rightarrow \infty$ as $n \rightarrow \infty$. This shows that $\phi(I(f)) \subset I(g)$.

For opposite inclusion, we note that if $z \in I(g)$, then we must have $\phi(z) \in I(g)$. As above,

$$
\phi\left(f^{n}(z)\right)=g^{n}(\phi(z)) \rightarrow \infty \text { as } n \rightarrow \infty .
$$

This shows that $z \in \phi(I(f))$ and so $I(g) \subset \phi(I(f))$. This proves that $\phi(I(f))=I(g)$. Remaining equality obtained from the facts $\partial I(f)=J(f)$ and $F(f)=\mathbb{C} \backslash J(f)$.

Proof of Theorem 1.1. Let $\phi \circ f_{i} \circ \phi^{-1}=g_{i}$ for all $i=1,2, \cdots, n$. From which we get $\phi \circ f_{i}=g_{i} \circ \phi$ for all $i=1,2, \cdots, n$. Any $f \in S$ and $g \in S^{\prime}$ can be written respectively as

$$
f=f_{i_{1}} \circ f_{i_{2}} \circ \ldots \circ f_{i_{n}}
$$

and

$$
g=g_{i_{1}} \circ g_{i_{2}} \circ \ldots \circ g_{i_{n}} .
$$

From which we get $\phi \circ f=\phi \circ f_{i_{1}} \circ f_{i_{2}} \circ \ldots \circ f_{i_{n}}=g_{i_{1}} \circ \phi \circ f_{i_{2}} \circ \ldots \circ f_{i_{n}}=g_{i_{1}} \circ g_{i_{2}} \circ \phi \circ \ldots \circ f_{i_{n}}=$ $\ldots=g_{i_{1}} \circ g_{i_{2}} \circ \ldots \circ g_{i_{n}} \circ \phi=g \circ \phi$ for all $f \in S$ and $g \in S^{\prime}$. Since $S=\left\langle f_{1}, f_{2}, f_{3}, \ldots, f_{n}\right\rangle$ be a nearly abelian holomorphic semigroup, so from [2, Theorem 1.1], we have

$$
I(S)=I(f), J(S)=J(f) \text { and } F(S)=F(f)
$$

for all $f \in S$.

$$
\text { Now, } I(S)=I(f) \Longrightarrow \phi(I(S))=\phi(I(f)) \text {. }
$$

By Lemma 2.1, $\phi(I(f))=I(g)$. By Theorem 2.1, semigroup $S^{\prime}$ is nearly abelian, so again by $\left[2\right.$, Theorem 1.1], we have $I\left(S^{\prime}\right)=I(g)$. Thus we get $\phi(I(S))=I\left(S^{\prime}\right)$. Next two equality are also obtained by the similar fashion.

\section{Proof of Theorem 1.2}

There is a nice way of writing arbitrary element of nearly abelian holomorphic semigroup. Before doing so, we see how does any $f \in S$ behave just like semi-conjugacy for some member of $\Phi(S)$ and a member from the set generated by $\Phi(S)$ as shown in the following lemma. Note that the statement and the proof this lemma is analogous to [1, Lemma 4.2]. 
Lemma 3.1. Let $S$ be a nearly abelian cancellative holomorphic semigroup. Then for any $f \in S$ and any $\phi \in \Phi(S)$, there is a map $\xi \in G$, where $G=\langle\Phi(S)\rangle$ is a group generated by the elements in $\Phi(S)$ such that $f \circ \phi=\xi \circ f$.

Proof. For any $\phi \in \Phi(S)$, there are $g, h \in S$ such that $g \circ h=\phi \circ h \circ g$. Then, for any $f \in S$, we can write

$$
f \circ g \circ h=f \circ \phi \circ h \circ g
$$

Furthermore,

$$
f \circ g \circ h=\xi_{1} \circ g \circ f \circ h=\xi_{1} \circ \xi_{2} \circ f \circ h \circ g .
$$

for some $\xi_{1}, \xi_{2} \in \Phi(S)$. Since $S$ is cancellative semigroup, so from the equations 3.1 and 3.2 , we get

$$
f \circ \phi=\xi_{1} \circ \xi_{2} \circ f=\xi \circ f
$$

where $\xi=\xi_{1} \circ \xi_{2} \in G$.

From the equation 3.2, we can say that composite of two commutators may not be a commutator. We investigate a couple of examples of holomorphic semigroups such that essence of above Lemma 3.1 holds.

Example 3.1. If a semigroup $S$ generated by functions $f(z)=\lambda \cos z \in S$ and $g(z)=$ $-f(z)$, then it is nearly abelian where $\phi(z)=-z$ is the commutator of $f$ and $g$ (see for instance [2, Example 2.2]). Since $\phi^{2}(z)=z$, an identity element of a group $G=\langle\Phi(S)\rangle=$ $\{$ Identity, $\phi\}$ such that $f \circ \phi=f=$ Identity $\circ f$ and $g \circ \phi=g=$ Identity $\circ g$.

Example 3.2. If a semigroup $S$ generated by functions $f(z)=e^{z^{2}}+\lambda$ and $g(z)=-f(z)$, then it is nearly abelian where $\phi(z)=-z$ is the commutator of $f$ and $g$ (see for instance [2, Example 2.2]). Since $\phi^{2}(z)=z$, an identity element of a group $G=\langle\Phi(S)\rangle=\{$ Identity, $\phi\}$ such that $f \circ \phi=f=$ Identity $\circ f$ and $g \circ \phi=g=$ Identity $\circ g$.

Also note that in both of these examples, we have $\phi \circ f=-f \neq f=f \circ \xi$ for any $\xi \in G$. That is, for given $\phi \in \Phi(S)$, there may not always possible to find element $\xi \in G$ satisfying $\phi \circ f=f \circ \xi$ for any choice of $\xi \in G$.

Proof of the Theorem 1.2. The proof of this theorem follows from the inductive application of Lemma 3.1 to each element $f=f_{i_{1}} \circ f_{i_{2}} \circ \ldots \circ f_{i_{n}}$ of $S$.

\section{REFERENCES}

[1] A. Hinkkanen, A., and G.J. Martin, The dynamics of semigroups of rational functions- I. Proc. London Math. Soc. 73(3)(1996): 358-384.

[2] B.H. Subedi, and A. Singh, A study of Fatou Set, Julia set and escaping set in nearly abelian transcendental semigroup. arXiv: 1808.00857v1[math. DS] (2018). 